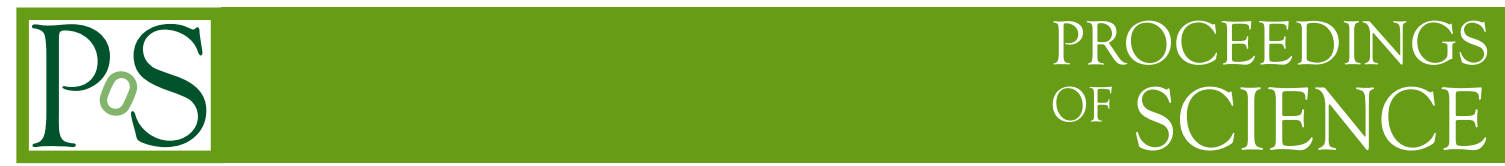

\title{
Rare Kaon Decay Experiments
}

\section{Taku Yamanaka*}

Osaka University, Toyonaka, Osaka 560-0043, JAPAN

E-mail: taku@champ.hep.sci.osaka-u.ac.jp

Rare kaon decays are sensitive probes to search for new physics beyond the standard model. This talk reviews the status of J-PARC KOTO and CERN NA62 experiments for the $K \rightarrow \pi v \bar{v}$ decays, J-PARC E36 for the lepton number universality, and CERN LHCb experiment for $K_{S}$ decays.

Flavor Physics and CP Violation,

6-9 June 2016

Caltech, Pasadena CA, USA

* Speaker. 


\section{Introduction}

Rare kaon decays are sensitive probes to search for new physics beyond the standard model. For example, kaon decays involving $s \rightarrow d$ quark transitions are suppressed, because they are allowed only through loop diagrams. Differences between the branching ratios of such decays and predictions of the standard model can signify contributions of new physics in the decays. In addition, kaons are suitable for studying rare processes because a large number of kaons can be produced simply by hitting a fixed target with protons.

\section{1 $K \rightarrow \pi v \bar{v}$ decay}

The $K \rightarrow \pi v \bar{v}$ decay modes are among the best probes to search for new physics [1]. In the standard model, the decay proceeds through a penguin diagram shown in Fig. 1. The standard model predicts their branching ratios to be $B R\left(K^{+} \rightarrow \pi^{+} v \bar{v}\right)=\left(8.22_{-0.65}^{+0.74} \pm 0.2\right) \times 10^{-11}$ and $B R\left(K_{L} \rightarrow \pi^{0} v \bar{v}\right)=\left(2.57_{-0.35}^{+0.38} \pm 0.04\right) \times 10^{-11}$ where the first error is due to CKM parameter measurements, and the second error summarizes theoretical uncertainties [2]. The small branching ratios and the small theoretical errors make the decays sensitive to contributions of new physics in these decays. In addition, because $\mathrm{CP}$ is violated in the $K_{L} \rightarrow \pi^{0} v \bar{v}$ decay, the decay is sensitive to new physics that breaks the $\mathrm{CP}$ symmetry. Various theoretical models predict the branching ratios to deviate by up to an order of magnitude $[3,4,5,6]$.

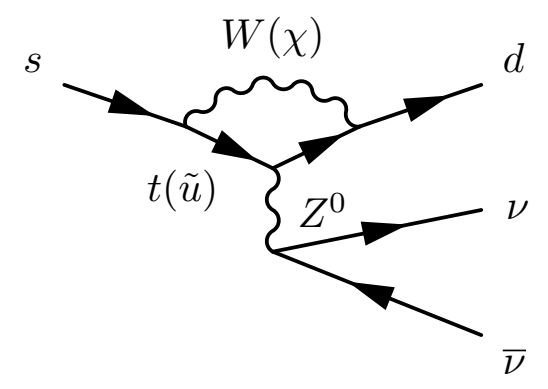

Figure 1: Penguin diagram of the $K \rightarrow \pi v \bar{v}$ decay.

The KEK E391a experiment set an upper limit on the branching ratio, $B R\left(K_{L} \rightarrow \pi^{0} v \bar{v}\right)<$ $2.6 \times 10^{-8}(90 \% \mathrm{CL})$ [7]. The BNL E787/E949 experiments measured $B R\left(K^{+} \rightarrow \pi^{+} v \bar{v}\right)=$ $\left(1.73_{-1.05}^{+1.15}\right) \times 10^{-10}$ based on 7 observed events [8]. This result indirectly gives an upper limit on the $K_{L} \rightarrow \pi^{0} v \bar{v}$ as $B R\left(K_{L} \rightarrow \pi^{0} v \bar{v}\right)<1.46 \times 10^{-9}(1 \sigma)$, called the "Grossman-Nir Limit" [9], but the limit does not apply to the $K_{L} \rightarrow \pi^{0} X^{0}$ decay where $X^{0}$ is a new-physics particle with the mass close to $m_{\pi^{0}}[10]$.

\section{J-PARC KOTO Experiment}

The J-PARC E14 KOTO is an experiment dedicated for the $K_{L} \rightarrow \pi^{0} v \bar{v}$ decay. A $K_{L}$ beam is made by collimating the $K_{L}$ s produced by $30-\mathrm{GeV}$ protons hitting a gold target. Figure 2 shows a schematic view of the KOTO detectors. The two photons from the $\pi^{0}$ decays are detected by an electromagnetic calorimeter made of 2716 undoped CsI crystals. To ensure that there are no other visible particles, the decay region is surrounded by hermetic photon veto and charged veto 
detectors. To minimize dead material between the $K_{L}$ vertex and the veto detectors, most of the detectors are placed in vacuum. All the signals from the detectors are digitized every $8 \mathrm{~ns}(2 \mathrm{~ns}$ for some detectors) to record their waveforms and to make trigger signals digitally.

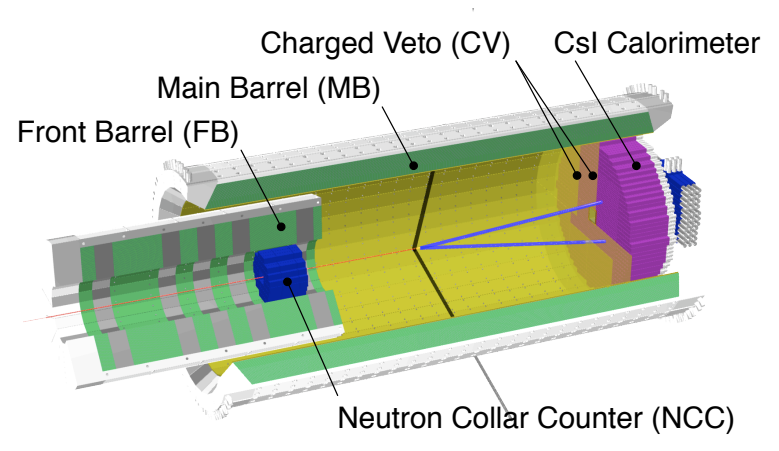

Figure 2: KOTO detector.

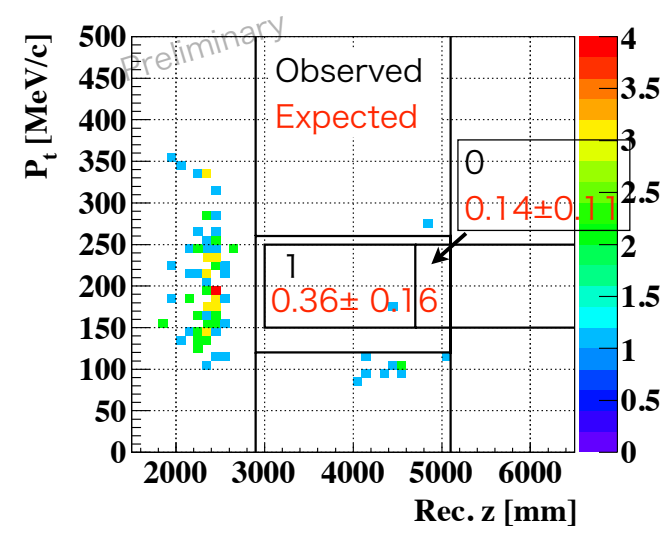

Figure 3: The $P_{T}$ and decay vertex positions of the events observed in the KOTO's first 4 days of run. The rectangular at the center shows the signal region. The numbers in black (red) show the numbers of observed (background) events.

The KOTO experiment took its first physics data in May 2013 for 100 hours with the proton beam power $24 \mathrm{~kW}$. The $K_{L}$ decay vertex was reconstructed by assuming that the two photons decayed from a $\pi^{0}$ on the beam axis. The $\pi^{0}$ 's momentum component normal to the beam axis, $P_{T}$, was reconstructed based on the decay vertex. Figure 3 shows the reconstructed $P_{T}$ and the decay vertex of the events observed in the data. Even with 4 days of data, the data had a sensitivity similar to that of E391a which ran for 6 months.

In 2015, the experiment collected 20 times more data than in 2013, aiming to cross the Grossman-Nir limit. In 2016, a new cylindrical photon veto detector was installed inside the existing detector to add 5 more radiation lengths $\left(X_{0}\right)$ to the existing 13.5 - $X_{0}$-thick cylindrical photon veto detector $(\mathrm{MB})$, and ran for 3 weeks with a $42 \mathrm{~kW}$ beam power.

In the 2013 run, one background event was observed. This event was consistent with a neutron directly hitting the calorimeter, producing two energy clusters. The KOTO group plans to suppress the neutron background by improving cuts on cluster shapes and signal pulse shapes. The group also plans to add solid-state photosensors (MPPCs) in front of CsI crystals to measure the shower depths which are different between photons and neutrons. With these improvements and by increasing the beam power from the current $42 \mathrm{~kW}$ to $80-100 \mathrm{~kW}$, the experiment should be able to search for new physics down to $B R<10^{-10}$.

\section{CERN NA62 Experiment}

The CERN NA62 experiment aims to measure the branching ratio of the $K^{+} \rightarrow \pi^{+} v \bar{v}$ decay with a $10 \%$ accuracy. It uses $K^{+} \mathrm{s}$ decaying in flight, unlike the BNL E787/949 experiments which used stopped kaons. The signal events are required to have 1) the square of the missing 
mass $M_{\text {miss }}^{2}=\left(E_{K}-E_{\pi}\right)^{2}-\left(\boldsymbol{p}_{K}-\boldsymbol{p}_{\pi}\right)^{2}$ away from that of other decays, 2) incoming and decayed particles identified as $K^{+}$and $\pi^{+}$, respectively, and 3) no photons associated with the decay, to suppress $K^{+} \rightarrow \pi^{+} \pi^{0}$ background.

Figure 4 shows a schematic view of the experiment. The incoming $75-\mathrm{GeV} / \mathrm{c} \mathrm{K}^{+} \mathrm{s}$ are tracked by three $300-\mu \mathrm{m}$-wide pixel detectors to achieve a $0.016 \mathrm{mrad}$ angle resolution and $200 \mathrm{ps}$ timing resolution. The decayed $\pi^{+}$tracks are detected by straw trackers with a position resolution of 140 $\mu \mathrm{m}$. With these resolutions, their measured preliminary missing mass resolution is close to the designed value as shown in Fig. 5.

The incoming $K^{+} \mathrm{s}$ are identified by a ring imaging Cherenkov counter and the track information. The data taken in 2015 shows that the counter can clearly select $K^{+} \mathrm{s}$ from charged pions in the high intensity beam. The decayed $\pi^{+} \mathrm{s}$ are identified by a ring imaging Cherenkov counter (pion RICH) filled with Neon gas. Muons are suppressed by 4-6 orders of magnitude by a liquid Ar calorimeter located downstream, and 2 orders of magnitude by the pion RICH counter [11].

Photons from the $K^{+} \rightarrow \pi^{+} \pi^{0}$ decays with large angles are detected by lead-glass counters arranged in a ring-shape (LAV) located at multiple locations. Photons with small angles are detected by the liquid $\mathrm{Kr}$ calorimeter (LKr). As shown in Fig. 6, the $\pi^{0}$ s from the $K^{+} \rightarrow \pi^{+} \pi^{0}$ decays were demonstrated to be suppressed by 5-6 orders of magnitude in the 2015 data.

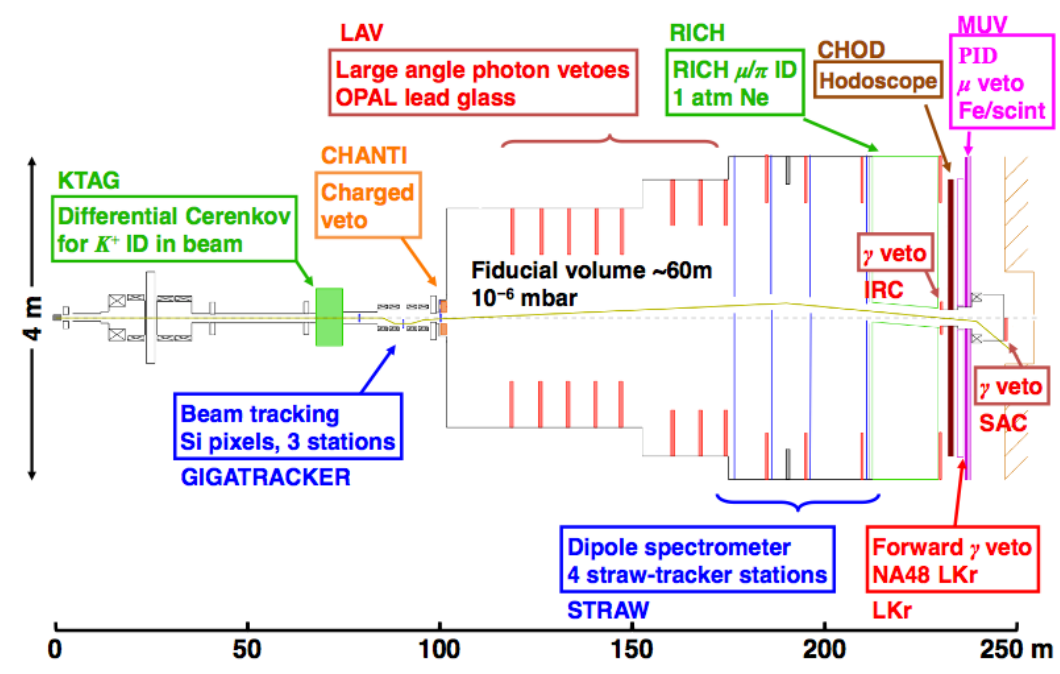

Figure 4: CERN NA62 detector [11].

The NA62 experiment has finished commissioning, and started taking data in April 2016. The experiment expects to collect $O(100)$ events by 2018 to measure the branching ratio with a $10 \%$ precision.

Besides the $K^{+} \rightarrow \pi^{+} v \bar{v}$ decays, the experiment studied other rare decays. The CERN NA48/2, the predecessor of NA62, searched for dark photons in the $K^{+} \rightarrow \pi^{+} \pi^{0}$ and $K^{+} \rightarrow \mu^{+} v \pi^{0}$ decays. It looked for a contribution of a dark photon coupled to a photon from $\pi^{0} \mathrm{~s}$ and then back to an $e^{+} e^{-}$pair. The obtained result filled a gap for the dark photon mass between 10 and $100 \mathrm{MeV} / c^{2}$ [14]. The NA62 also measured a $\pi^{0}$ transition form factor which is a function of $e^{+} e^{-}$mass in the $\pi^{0}$ Dalitz decay. This form factor gives an input to the calculation of "light-by-light" correction for the theoretical calculation of $g-2$. Their preliminary result, $a=(3.70 \pm 0.53 \pm 0.36) \times 10^{-2}$ 


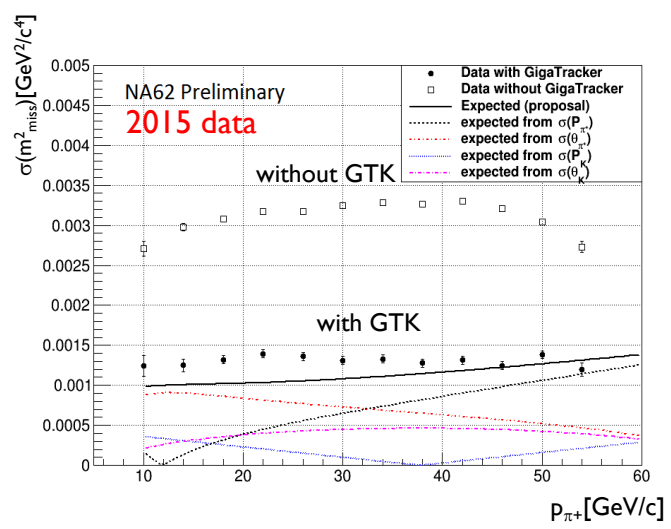

Figure 5: The NA62's resolution of $M_{\text {miss }}^{2}$ as a function of the $\pi^{+}$momentum. The black points show the preliminary measured in the 2015 data, and the black line shows the expected resolution [12].

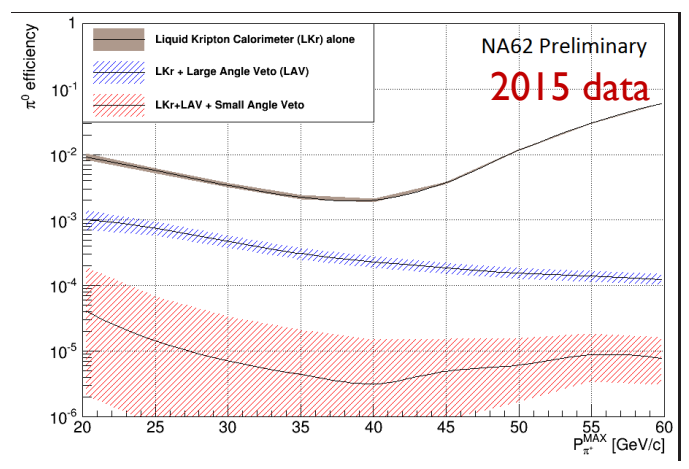

Figure 6: NA62's $\pi^{0}$ detection inefficiency as a function of $P_{\pi^{0}}^{\max }$, measured with $K^{+} \rightarrow \pi^{+} \pi^{0}$ decay events collected in 2015 [12].

[13] is 5 times more accurate than the past best measurement. The NA62 also plans to improve the limits on lepton number and flavor violating decay modes by 1-2 orders of magnitude. The NA62 tested lepton universality by measuring the ratio $R_{K}=\Gamma\left(K^{+} \rightarrow e^{+} v\right) / \Gamma\left(K^{+} \mu^{+} v\right)=$ $(2.488 \pm 0.010) \times 10^{-5}[15]$.

\section{J-PARC TREK-E36 Experiment}

The J-PARC TREK-E36 experiment ran in 2015 to measure lepton number universality. Figure 7 shows the E36 detector. A $K^{+}$beam was stopped in a target of scintillating fibers, and the momenta of decayed muons and electrons were measured. The decayed particles were identified by aerogel Cherenkov counters surrounding the target, and lead glass counters placed at the exit of the spectrometer. The experiment collected $40 \mathrm{k} K^{+} \rightarrow e^{+} v$ decays, and expects to measure the ratio $R$ with a $0.5 \%$ error.

\section{CERN LHCb Experiment}

The CERN LHCb experiment was designed to study $B$ decays, but it is also a $K_{S}$ factory. For example, the experiment searched for the $K_{S} \rightarrow \mu^{+} \mu^{-}$decays as a signature for new physics. The standard model predicts $B R_{\mathrm{SM}} \sim 5 \times 10^{-12}$ [18], and new physics could enhance the branching ratio. The experiment observed events with the yield consistent with background expectation, and measured $B R\left(K_{S} \rightarrow \mu^{+} \mu^{-}\right)<9 \times 10^{-9}$ (90\% CL) [17], improving the best upper limit on the branching ratio measured in 1973 by a factor 30 . The experiment is expected to increase the statistics by a factor 30 by use of an integrated luminosity, and possibly another factor 3 by trigger improvement; the improvement on the limit depends on the background reduction. 


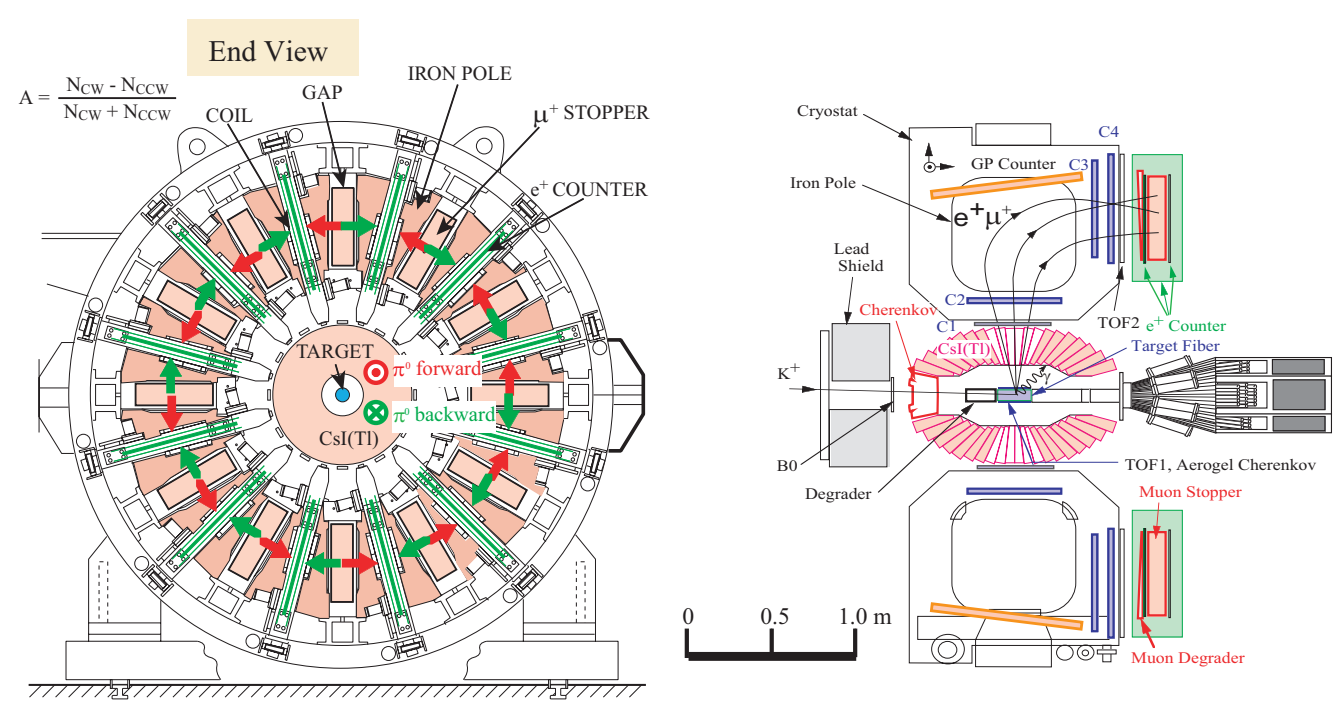

Figure 7: J-PARC E36 detector [16].

\section{Summary}

Rare kaon experiments are exploring new physics beyond the standard model in the 10-10 $\mathrm{TeV}$ energy scale via the $K \rightarrow \pi v \bar{v}$ decay modes, and even higher energy scale via lepton flavor violation.

\section{Acknowledgement}

I would like thank A. Ceccucci, S. Shimizu, and A. Puig for supplying information.

\section{References}

[1] L.S. Littenberg, CP-violating decay $K_{L} \rightarrow \pi^{0} v \bar{v}$, Phys. Rev. D 39 (1989) 3322.

[2] J. Brod, M. Gorbahn, and E. Stamou, Two-loop electroweak corrections for the $K \rightarrow \pi v \bar{v}$ decays, Phys. Rev. D 83 (2011) 034030.

[3] M. Blanke et al., Rare and CP-violating $K$ and B decays in the Littlest Higgs model with T-parity, JHEP 01 (2007) 066.

[4] A.J. Buras et al., Can we reach the Zeptouniverse with rare $K$ and $B_{s, d}$ decays?, JHEP 11 (2014) 121.

[5] M. Tanimoto and K. Yamamoto, $K_{L} \rightarrow \pi^{0} v \bar{v}$ decay correlating with $\varepsilon_{K}$ in high-scale SUSY, Prog. Theor. Exp. Phys. 2015 (2015) 053B07.

[6] A.J. Buras, D. Buttazzo, and R. Knegjens, $K \rightarrow \pi v \bar{v}$ and $\varepsilon^{\prime} / \varepsilon$ in simplified new physics models, $J$. High Energ. Phys. 2015 (2015) 166.

[7] J.K. Ahn et al., Experimental study of the decay $K_{L} \rightarrow \pi^{0} v \bar{v}$, Phys. Rev. $D 81$ (2010) 072004.

[8] A.V. Artamonov et al., New Measurement of the $K^{+} \rightarrow \pi^{+} v \bar{v}$ Branching Ratio, Phys. Rev. Lett. 101 (2008) 191802.

[9] Y. Grossman and Y. Nir, $K_{L} \rightarrow \pi^{0} v \bar{v}$ beyond the Standard Model, Phys. Lett. B 398 (1997) 163. 
[10] K. Fuyuto, W.-S. Hou, and M. Kohda, Loophole in $K \rightarrow \pi v \bar{v}$ Search and New Weak Leptonic Forces, Phys. Rev. Lett. 114 (2015) 171802.

[11] M. Mirra, Search for the $K^{+} \rightarrow \pi^{+} v \bar{v}$ at the NA62 experiment at CERN, presented at HQL2016, Virginia, USA (2016).

[12] F. Bucci, NA62 Results and Prospects, presented at BEAUTY 2016, Marseille, France (2016).

[13] M. Koval, Results and Perspectives from the NA62 Experiment at CERN, presented at Les Recontres de Physique de la Vallée d'Aoste, La Thuile, Italy (2016).

[14] J.R. Batley et al., Search for the dark photon in $\pi^{0}$ decays, Phys. Lett. B 746 (2015) 178.

[15] C. Lazzeroni et al., Precision measurement of the ratio of the charged kaon leptonic decay rates, Phys. Lett. B 719 (2013) 326.

[16] J-PARC TREK Collaboration, Measurement of $\Gamma\left(K^{+} \rightarrow e^{+} v\right) / \Gamma\left(K^{+} \rightarrow \mu^{+} v\right)$ and Search for heavy sterile neutrinos using the TREK detector system, KEK J-PARC-PAC2010-04 (2010) .

[17] R. Aaij et al., The LHCb Collaboration, Search for the rare decay $K_{S}^{0} \rightarrow \mu^{+} \mu^{-}$, J. High Energ. Phys. 01 (2013) 090.

[18] G. Isidori and R. Unterdorfer, On the short-distance constraints from $K_{L, S} \rightarrow \mu^{+} \mu^{-}$, J. High Energ. Phys. 01 (2004) 009. 